American J. of Engineering and Applied Sciences 3 (2): 390-395, 2010

ISSN 1941-7020

(C) 2010 Science Publications

\title{
Full Model Wind Tunnel Study on the Xia-Zhang Bridge Under Operation Stage
}

\author{
Ayad Thabit Saeed, Zhang Liang Liang, Yang Zhuan Yun, \\ Feras Al Zoubi and Oday Asal Salih \\ Department of Bridge and Tunnel Engineering, College of Civil Engineering, \\ Chongqing University, Chongqing City-400045, China
}

\begin{abstract}
Problem statement: Long-span cable-stayed bridges under service and particular construction conditions are very susceptible to wind action due to their great flexibility, so the aerodynamic stability is becoming a major concern in the design and construction phrases. Cablestayed bridges may exhibit wind-induced vibration phenomena such as flutter, buffeting and vortex oscillation under wind excitation. The study concentrated on the issues concerning the aerodynamic response of Xia-Zhang cable-stayed Bridge to make it safe and stable under wind action. Although there have been accumulating experience in the building of cable-stayed bridges and research on windresistant stability in Chinese Mainland, most of the research focuses on inland cable-stayed bridges or littoral ones of mid-length, but not on littoral ones whose main span is over $600 \mathrm{~m}$. Therefore, windresistant performance research of north branch bridge of Xia-Zhang cross-sea Bridges is very necessary and important for its wind-resistant stability, safety and applicability in the operation condition. Approach: This study mainly presented the wind tunnel test program of the Xia-Zhang Bridge aeroelastic full model, including test method, test contents, test results and so on. Results: The test results contained Root Mean Square (RMS) of accelerations and displacements as well as average values of displacements. Conclusion: The conclusions were as follows: (a) In the uniform flow field, under the condition of entire bridge without rail may vibration divergence occur when $\alpha=3^{\circ}, \mathrm{V}>122$ $\mathrm{m} \mathrm{sec}^{-1}$. (b) No vortex-induced vibration with extreme amplitudes or static collapsing was detected in all the testing conditions. (c) In the turbulent flow field, there were very obvious buffeting phenomena. Responses to turbulence are quite intense.
\end{abstract}

Key words: Xia-Zhang Bridge, aeroelastic model, wind tunnel test

\section{INTRODUCTION}

Xia-Zhang cross-sea Bridges are located to the east of Xiamen city and Zhangzhou city in China, across the access to the Sea of Jiulong River. Among them, the bridge that connects Haichang Borough and Haimen Island is called North Branch Bridge, which is 6392.6 $\mathrm{m}$ in length. The bridge contains three parts (Low speed Institute of CARDC, 2007; Saeed et al., 2010; Wang, 2008):

- The main bridge, which is a cable-stayed bridge combined with steel and concrete, $1290 \mathrm{~m}$ in length. And its main span is $720 \mathrm{~m}$ in length

- The north approach bridge, whose main span is $1130 \mathrm{~m}$ in length

- The south approach bridge, whose main span is $3972.6 \mathrm{~m}$ in length
The length of the main span of the main bridge $(720 \mathrm{~m})$, ranks No. 6 world-wide among its category (cable-stayed bridge combined with steel and concrete) and ranks No. 4 among littoral ones in its category. The first 3 ones are: Angchuanzhou Bridge in Hong Kong, main span at $1018 \mathrm{~m}$ in length; Tatara Bridge in Japan, main span at $890 \mathrm{~m}$ in length; and Normandy Bridge in France, main span at $856 \mathrm{~m}$ in length (Holmes, 2007; Miyata, 2003; Taly, 1998; Xiang, 2005).

The wind tunnel test was proposed by the Civil Engineering College of Chongqing University and approved by the China Aerodynamics Research and Development Center (CARDC) (Haan, 2000; Low speed Institute of CARDC, 2007; Wang, 2008).

According to the previous analysis and results of partial model wind tunnel testing, a wind tunnel test of entire bridge aeroelastic model of North Branch Bridge of Xia-Zhang Cross-sea Bridges was proposed (Low speed Institute of CARDC, 2007; Wang, 2008). The

Corresponding Author: Ayad Thabit Saeed, Department of Bridge and Tunnel Engineering, College of Civil Engineering, Chongqing University, Chongqing City-400045, China Tel: 0086-15909320090 
contents of the work in this wind tunnel test are as follows:

- Design and manufacturing of the entire bridge aeroelastic model of North Branch Bridge. This model includes: The model of stay-cable, the model of the main girder, the model of the main tower, the assistant pier, the transitional pier and the model of the restriction

- Simulation of atmospheric boundary layer flow field. The ground surface roughness level is determined as a type (A) ground, roughness coefficient $\alpha$ is set to be 0.12 and the height of gradient wind is set to be $300 \mathrm{~mm}$ (Xiang, 2005)

- Wind Tunnel Test of the Entire Bridge Aeroelastic Model:

- Tests for vortex-induced vibration. Vortexinduced vibration phenomena observation in operation condition and critical wind speed measurement

- Tests for buffeting response. Tests for buffeting response of different edge angle, attack angles, wind speeds in operation condition

\section{Model and equipment:}

Model: Five dimensionless constants are required as working conditions in the design of the entire bridge aeroelastic model. They are: Reynolds number, Froude number, density ratio, Cauchy number and critical damping ratio. Like most wind-tunnel tests, this test could not perfectly fulfill the similarity of Reynolds Number. However, research indicates that it is not important because the similarity in appearances of the detouring flow around the bridge is not remarkably affected in the tests for bluff bodies like bridges (especially for trusses).

Similarities in elastic, mass and gravity parameters (Froude number, density ratio and Cauchy number) are all strictly required. Similarity in Critical Damping Ratio is very difficult to acquire from the design of the model, but conclusions of previous experiences indicate that the Critical Damping Ratios of aeroelastic models are usually lower than the actual values, which emphases safety (Haan, 2000; Rousseau, 2004).

The geometric scaled ratio of the entire bridge aeroelastic model of North Branch Bridge of Xia-Zhang Cross-sea Bridges is $C_{L}=1: 150$. Based on the laws of model similarity, parameters could be calculated as follows: wind speed ratio $C_{v}=1: 12.25$, frequency ratio $\mathrm{C}_{\mathrm{f}}=12.25$, linear acceleration $\mathrm{C}_{\mathrm{av}}=1$, torsional acceleration $\mathrm{C}_{\mathrm{aT}}=150$ and so on. Detailed model designing parameters and achieved values are shown in Table 1.

For the main girder, a form of core girder combined with aerodynamic outer frame is adopted in the manufacturing of the model. The core girder is made of steel, which would fulfill the requirements of similarities in stiffs of vertical, lateral bending and free torsion. The mass of the main girder is composed of the core girder, outer frame and counterweights. The mass and the position of the counterweights could be adjusted to fulfill the requirements of the laws of model similarity. The bridge tower is manufactured in the same way as the main girder, where counterweights are also used (Wang, 2008). Figure 1 shows the model installed in the wind tunnel.

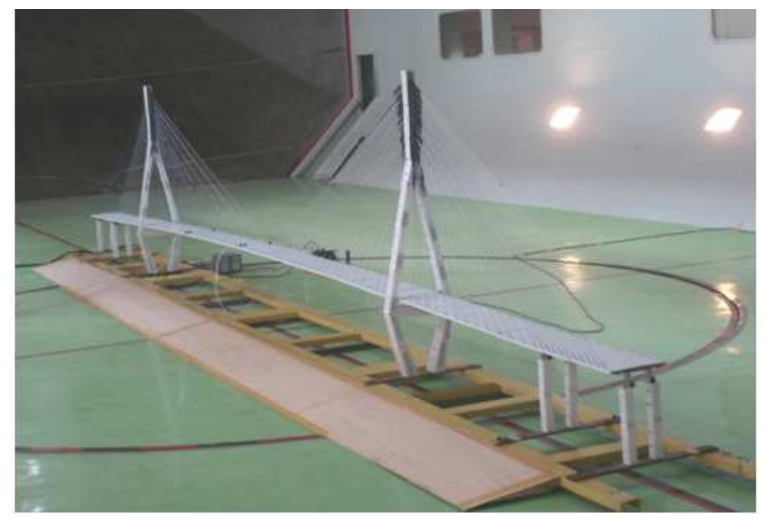

Fig. 1: The picture of the installed entire bridge model in the wind tunnel under uniform flow field

Table 1: Parameters of the entire bridge model and achieved values

\begin{tabular}{|c|c|c|c|c|c|c|}
\hline Parameters values & Symbol & Units & Scale & Actual values & Designed values & Achieved \\
\hline Length & $\mathrm{L}$ & $\mathrm{m}$ & $1: 150$ & 1290 & 8.60000 & 8.60000 \\
\hline Width & $\mathrm{B}$ & $\mathrm{m}$ & $1: 150$ & 38 & 0.25300 & 0.25300 \\
\hline Height & $\mathrm{H}$ & $\mathrm{m}$ & $1: 150$ & 3.5 & 0.02300 & 0.02300 \\
\hline Mass & $\mathrm{m}$ & $\mathrm{Kg} \mathrm{m}^{-1}$ & $1: 150^{2}$ & 32412 & 1.44000 & 1.44000 \\
\hline Inertia moment per unit mass & $\mathrm{I}_{\mathrm{m}}$ & $\mathrm{Kg} \cdot \mathrm{m}^{2} \mathrm{~m}^{-1}$ & $1: 150^{4}$ & 3245192 & 0.00641 & 0.00641 \\
\hline First-order frequency of vertical bending & $\mathrm{f}_{\mathrm{h}}$ & $\mathrm{Hz}$ & $\sqrt{150}: 1$ & 0.264 & 3.23200 & 3.25000 \\
\hline First-order frequency of twisting & $\mathrm{ft}$ & $\mathrm{Hz}$ & $\sqrt{150}: 1$ & 0.684 & 8.37800 & 8.25000 \\
\hline Vertical Stiffness of the main girder & $\mathrm{E} \mathrm{I}_{\mathrm{x}}$ & $\mathrm{Pa} \times \mathrm{m}^{4}$ & $1: 150^{5}$ & $7.836 \mathrm{E} 11$ & 10.31900 & 10.32000 \\
\hline Lateral stiffness of the main girder & $\mathrm{E} \mathrm{I}_{\mathrm{y}}$ & $\mathrm{Pa} \times \mathrm{m}^{4}$ & $1: 150^{5}$ & $5.1551 \mathrm{E} 13$ & 678.85000 & 678.90000 \\
\hline Twisting stiffness of the main girder & GJ & $\mathrm{Pa} \times \mathrm{m}$ & $1: 150^{5}$ & 8.5092E11 & 11.21000 & 11.20000 \\
\hline Damping ratio & $\zeta$ & - & $1: 1$ & - & - & $0.61000 \%$ \\
\hline
\end{tabular}


Wind tunnel: The test is carried out in a large, lowspeed wind tunnel of $8 \times 6 \mathrm{~m}$. This wind tunnel is a direct, closing tunnel with double tandem test sections. The profile of the first section is an angle-cut rectangle of $12 \times 16 \mathrm{~m}$. This section is $25 \mathrm{~m}$ in length and the stable wind speeds in it could be $1.0-18.0 \mathrm{~m} \mathrm{sec}^{-1}$. This section is installed with spire, fence and roughness elements for simulating the atmospheric boundary layer, which generates a wind environment defined by "Design Specification of Bridge Aiming Wind Resistance" and "construction structure load design specification" (Saeed et al., 2010; Wang, 2008). The roughness coefficient is set to be 0.12 .

Measuring equipment: Measuring equipments for this wind tunnel test include: FocusII dynamic signal collecting and analyzing system, acceleration sensor, hot wire anemometer (IFA300), dynamic displacement binocular measuring system and pitot tube. Signals as vibration acceleration, displacement, were real-timely collected, displayed and stored in a dynamic signal collecting and analyzing system in the test.

\section{MATERIALS AND METHODS}

To investigate the similarities between the model and the prototype, modal tests were carried out after the installation of the model in the wind tunnel. The hammering method is used in the modal test. The testing system contains acceleration sensors and Focus II dynamic signal collecting and analyzing system. Structure vibration spectrum could be acquired realtimely by the test system. Subsequently, the structure damping ratio could be calculated from vibration free damped duration with the following formula (Huang, 2006; Saeed et al., 2010; Wang, 2008):

$\xi=\frac{\delta_{\mathrm{n}}}{2 \pi \cdot \mathrm{n}}$

where, $\delta_{n}=\operatorname{In}\left(\frac{A_{i}}{A_{i+n}}\right)$ and $A_{i}, A_{i+n}$ means amplitudes of cycle $i$ and $i+n$. The results of this test are shown in Table 1 . The achieved main vibration frequency of the model is very close to the required value and the achieved modal damping ratio is among the required range. So the model can meet the requirements.

Flow field examinations: Flow field of type (A) atmospheric boundary layer are simulated before the test. A passive simulation using devices like spires and roughness elements were adopted. A hot wire anemometer was also used to examine the simulation. The required flow field could be achieved by adjusting the density of spires and roughness elements. Figure 2 shows the picture of simulating the boundary layer flow field in this test. Figure 3 shows the wind speed section and turbulence section of the flow field. The results of the test indicate that the simulated wind speed section index is 0.11943 , very close to the theoretic value 0.12 . So the simulated turbulent flow field can meet the requirement (Saeed et al., 2010; Wang, 2008).

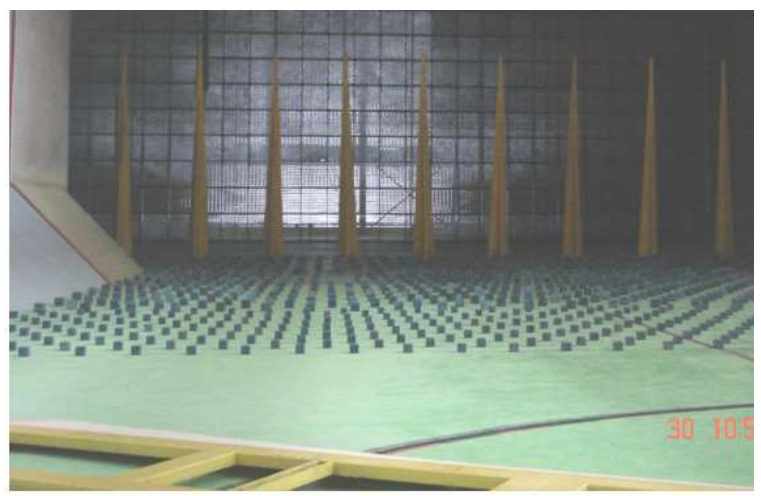

Fig. 2: The flow field simulation picture of atmospheric boundary layer type (A)

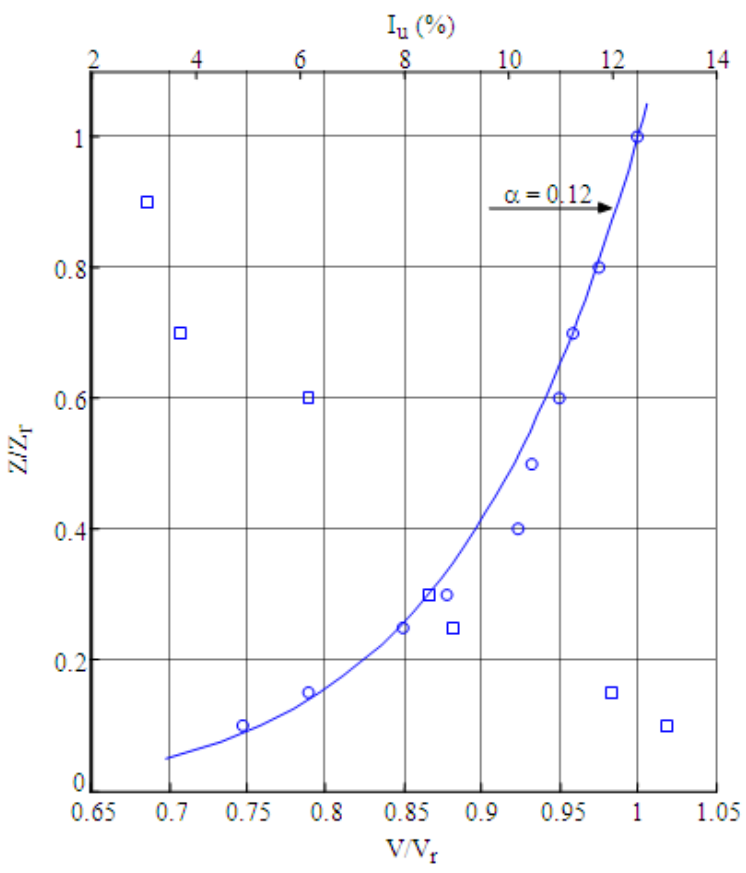

Fig. 3: The wind speed section and turbulence section of simulated flow field 
Am. J. Engg. \& Applied Sci., 3 (2): 390-395, 2010

Table 2: The wind tunnel test contents of Xia-Zhang bridge model

\begin{tabular}{lllll}
\hline Flow field & Model & Wind speed $\left(\mathrm{m} \mathrm{sec}^{-1}\right)$ & Attack angle $\alpha\left(^{\circ}\right)$ & Wind angle $\beta\left(^{\circ}\right)$ \\
\hline Turbulent flow field & Entire bridge with rail & $2-8 \Delta \mathrm{V}=0.5$ & $\alpha=-3,0,3$ & $\beta=0,22.5,45,67.5,90$ \\
Uniform flow field & Entire bridge with rail & & $\alpha=0,3$ & $\beta=0,45$ \\
Uniform flow field & Entire bridge without rail & $2-10 \Delta \mathrm{V}=0.5$ & $\alpha=-3,0,3$ & $\beta=0,10,20,30,45,60,90$ \\
\hline
\end{tabular}

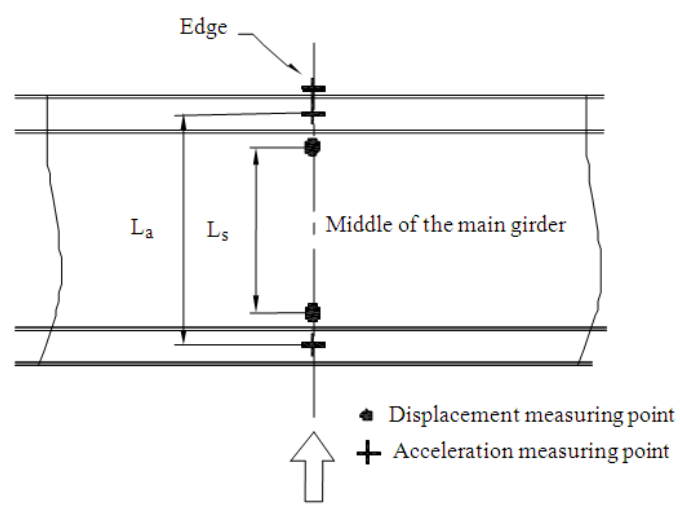

Fig. 4a: Measure points layout picture

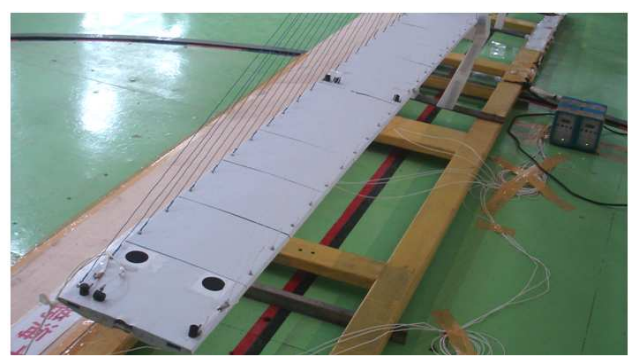

Fig. 4b: Layout picture of measure points in the main girder

Test contents: Tests of aeroelastic model of north branch bridge of Xia-Zhang cross-sea Bridges were carried out under the entire bridge condition (operation condition). The Displacements and accelerations at the top of the unique tower, the center of the main girder and the 1/4 location of the main span were measured in the test. Uniform flow field and turbulent flow field were both used in the test. Wind angle $\beta$ could be changed by rotating the rotary table of the wind tunnel. Wind angle $\beta$ is set to be $0^{\circ}$ when the wind flows against the lateral face of the model. Attack angle should be $0^{\circ}, \pm 3^{\circ}$. One side of the model was blocked up to become lean so that attack angle of $\pm 3^{\circ}$ could be attained. And the inflow speed was examined by the pitot tube. The detailed test contents are shown in Table 2.

Measure point arrangement and data processing: Displacements and acceleration response surveys: Locations of where to measure displacements: The top of the unique tower (in the directions of across and along the bridge); the $1 / 4$ and $1 / 2$ locations of the main girder under entire bridge condition (in the directions of across the bridge, vertical and twisting). The displacements were measured by the dynamic displacement binocular measuring system.

The locations of to measure accelerations were the same with the displacements as well as the directions. The accelerations were measured by acceleration sensors. Figure $4 \mathrm{a}$ and $\mathrm{b}$ shows the measuring points in the main girder. The displacements in the vertical directions $S_{v}$ and the displacements in the twisting direction $S_{t}$ in the main girder could be calculated by the following formulas:

$$
\begin{aligned}
& \mathrm{S}_{\mathrm{v}}=\left(\mathrm{S}_{\mathrm{a}}+\mathrm{S}_{\mathrm{b}}\right) \mid 2 \\
& \mathrm{~S}_{\mathrm{t}}=\left(\mathrm{S}_{\mathrm{a}}-\mathrm{S}_{\mathrm{b}}\right) \mid \mathrm{L}_{\mathrm{a}}
\end{aligned}
$$

Where:

$$
\begin{aligned}
& \mathrm{S}_{\mathrm{a}}, \mathrm{S}_{\mathrm{b}}=\text { Displacements of points (a) and (b) } \\
& \mathrm{L}_{\mathrm{a}}=\text { The distance between the points }
\end{aligned}
$$

The accelerations in the vertical directions and the accelerations in the twisting direction could be calculated in the same way.

\section{RESULTS}

The test results of aeroelastic model of north branch bridge of Xia-Zhang cross-sea Bridges were carried out under the uniform flow field and turbulent flow field. The Root Mean Square (RMS) of Displacements and accelerations at the top of the tower, the center of the main girder and the 1/4 location of the main span as well as average values of displacements were measured in the test. Wind angle $\beta$ could be changed from $0^{\circ}$ to $90^{\circ}$ Attack angle should be $0^{\circ}, \pm 3^{\circ}$ as shown in Table 2. Moreover all the results have already been transformed to actual values (Kim et al., 2009; Zhao and Ge, 2009).

Figure 5 shows the comparison of the RMS of accelerations in the twisting direction at the $1 / 4$ location of the main span in the entire bridge with rail under the uniform flow field when $\alpha=0^{\circ}$ in different attack angles. 


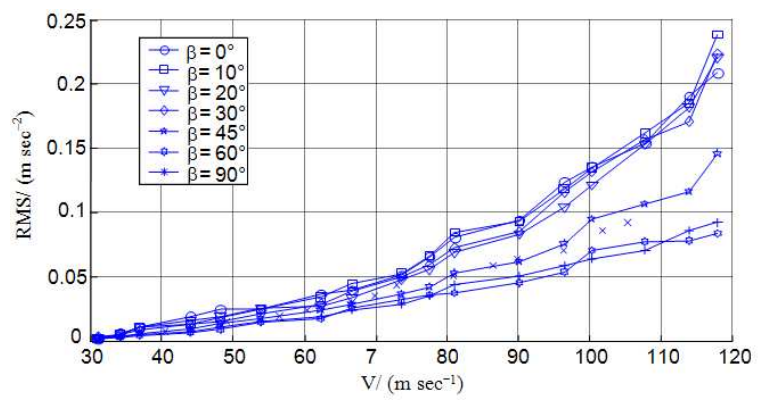

Fig. 5: The RMS of accelerations in the twisting direction at the 1/4 location of the main span in the entire bridge with rail under the uniform flow field when $\alpha=0^{\circ}$

\section{DISCUSSION}

Uniform flow experiments: No vortex-induced vibration with extreme amplitudes was detected in all the tested wind speeds. Under the condition of entire bridge without rail, no vibration divergence was detected except when $\alpha=3^{\circ}, \mathrm{V}>120 \mathrm{~m} \mathrm{sec}^{-1}$. The vibration divergence is the reason for vibration destabilization.

As in the results of RMS of accelerations obtained after adding rails, accelerations in vertical and twisting directions of the $1 / 4$ and $1 / 2$ locations of the main girder decreased. But accelerations in the direction of across the bridge of the $1 / 2$ locations of the main girder and accelerations in the direction of across the bridge of the top of the tower clearly increased. When the actual wind speed is $92 \mathrm{~m} \mathrm{sec}^{-1}$, the maximum RMS is about 0.3 .

Under the same attack angle, accelerations decrease when $\beta$ increases. But when $\beta=90^{\circ}$, accelerations in the direction of across the bridge of the top of the tower become higher again. Accelerations do not change substantially when the attack angle increases from $3-3^{\circ}$.

As in the results of RMS of displacements obtained when $\alpha=-3^{\circ}$ and $\alpha=0^{\circ}$, the RMS of displacements are all very small. But vibration in vertical directions of the $1 / 2$ location of the main girder is comparatively a little higher and it decreases when $\beta$ increases.

When $\alpha=3^{\circ}$, the RMS of displacements in vertical and twisting directions increase rapidly when wind speed increases and decrease when $\beta$ increases. Figure 5 shows the comparison of RMS of accelerations of some points in different attack angles and the same wind angle.

Turbulent flow experiments: No vortex-induced vibration with extreme amplitudes was detected in all the testing wind speeds (actual wind speed $\mathrm{V} \leq 98 \mathrm{~m}$ $\sec ^{-1}$ ), but there were very obvious buffeting phenomena. The results of the RMS of vibration accelerations show that, under the same attack angle, the RMS of accelerations decreases slightly when $\beta$ increases and accelerations do not change substantially when the attack angle increases from $3-3^{\circ}$.

The results of the RMS of displacements show that, the RMS of displacements in the direction of across and along the bridge of the top of the tower increases rapidly. The RMS of displacements in the twisting direction of the center of the main girder is also very high, with the maximum one reaching $0.3^{\circ}$, but after $\beta>45^{\circ}$, it becomes smaller.

\section{CONCLUSION}

- For the Xia-Zhang Cross-sea Bridges, the basic wind speed was designed as $49.5 \mathrm{~m} \mathrm{sec}^{-1}$ and the critical flutter wind speed were $72.2 \mathrm{~m} \mathrm{sec}^{-1}$. After conversion, the actual wind speed of the entire bridge model was between $24.5 \mathrm{~m} \mathrm{sec}^{-1}$ and $98 \mathrm{~m}$ $\mathrm{sec}^{-1}$ and the actual wind speed of the unique tower model was between $49 \mathrm{~m} \mathrm{sec}^{-1}$ and $159 \mathrm{~ms} \mathrm{ec}^{-1}$. In both of the conditions above, no obvious flutter, buffeting or excitation was detected. In the uniform flow field, under the condition of the entire bridge without rail, the vibration divergence was detected when $\alpha=3^{\circ}, \mathrm{V}=122 \mathrm{~m} \mathrm{sec}^{-1}$

- In both the uniform and turbulent flow fields, no flutter or static collapsing and no vortex-induce vibration with extreme amplitude was detected in all the test

- In the turbulent flow field, there were very obvious buffeting phenomena in all $\beta$ angle ranges when the attack angle was $-3^{\circ}$ and the testing wind speed got higher than $36.7 \mathrm{~m} \mathrm{sec}^{-1}$. Responses to turbulence were quite intense.

\section{REFERENCES}

Haan, F.L., 2000. The effects of turbulence on the aerodynamics of long-span bridges. Ph.D. Thesis, University of Notre Dame, Notre Dame, Indiana. http://www.nd.edu/ nathaz/journals/HaanDissertati on2000.pdf

Holmes, J., 2007. Wind Loading Structures. 2nd Edn., Tayler and Francis, ISBN: 0-203-96428-4, pp: 433.

Huang, H.J., 2006. Test report for the aeroelastic model of baling river bridge in operation and construction process conditions. Low Speed Institute of CARDC, China. 
Kim, S.H., J.T. Park and K.J. Lee, 2009. The study of aerodynamic stabilizing for tangential and curved cable-stayed bridge under construction. Proceeding of the 7th Asia-Pacific Conference on Wind Engineering, Taipei, Taiwan, pp: 4. http://140.112.208.155/apcwe/PAPERS/10148.pdf

Low speed Institute of CARDC, 2007. Wind tunnel outline for the models of the Xia-Zhang Bridge. China.

Miyata, T., 2003. Historical view of long-span bridge. Aerodynamics. J. Wind Eng. Ind. Aerodyn., 91: 1393-1410. DOI: 10.1016/j.jweia.2003.09.033

Rousseau, G., 2004. Wind-induced dynamic response of bridges. Master of Engineering Thesis, Massachusetts Institute of Technology. http://hdl.handle.net/1721.1/29416

Saeed, A.T., L.L. Zhang, A. Z. Feras, O.A. Salih and H. Mohammad Agha, 2010. Wind tunnel study on the Xia-Zhang bridge under construction: Unique tower condition. Proceeding of the 3rd International Earthquake Symposium, Dhaka, Bangladesh.
Taly, N., 1998. Design of Modern of Highway Bridges. McGraw-Hill Company, Inc., New York, USA., ISBN: 0-07-062997-8 p. 1352.

Wang, W.H., 2008. Wind tunnel test report for the models of the Xia-Zhang Bridge: The part of section models. Low Speed Institute of CARDC, China.

Xiang, H.F., 2005. Wind Theory and Practice of Modern Bridges. People's Transportation Press, Beijing, China, ISBN: 7114058217, pp: 450.

Zhao, L. and Y.J. Ge, 2009. Buffeting response sensitivity of multi-component aerodynamic admittance function of typical bridge deck. Proceeding of the 7th Asia-Pacific Conference on Wind Engineering, Taipei, Taiwan, pp: 4. http://140.112.208.155/apcwe/PAPERS/10200.pdf 